\title{
Fungal Granuloma of long bone in immune-competent host .
}

\author{
Dr Sandesh S. Thete, Dr Satish Goyal
}

\begin{abstract}
:
Introduction

Increased incidence of fungal infection has been reported globally in the recent years. This has been attributed to increased aging population and longer survival of immune-compromised people. But granulomatous affection of bone is a rare and unusual presentation. Osteoarticular involvement may occur either by contiguous spread through a cutaneous focus, through direct inoculation of tissue by the organism, or by hematogenous dissemination.

Case report: This paper reports one of the very few known occurrence of a primary fungal granuloma in one of the long bones in a healthy individual with no evidence of any other primary fungal infection. Also we have discussed the treatment of this rare affection of bone.

Conclusion: Diagnosis of fungal infections requires a high index of suspicion. The text also emphasizes the importance of including endemic fungal infections in the differential diagnosis of bone lesions.
\end{abstract}

Keywords: Fungal infections; immune-competent host; long bone; granuloma

\section{Introduction}

Fungi have emerged as significant pathogens only during the past few decades. Currently the number of fungal species reported as being opportunistic agents of human disease is increasing by about 10 to 15 per year. Possible explanations are the general availability of broad-spectrum parenteral antibiotics, which results in fungal superinfections [1], and an increasing number of immuno-compromised patients. Actinomycosis, Nocardiosis, Cryptocosis, Histoplasmisis and aspergillosis are few etiological agents to mention. Aspergillus osteomyelitis usually results from direct invasion from a contiguous infection such as a pulmonary or nasal sinus abscess, and it almost always involves the ribs, spine, or orbit [2,3]. Although secondary destruction of long bones by Aspergillus has been occasionally described, in very few cases did the bones show primary involvement [3]. We report a case of primary fungal infection of long bone caused by aspergillus in an immunecompetent host.

\section{Case report}

A 50 year old male presented to the orthopedic OPD with history of pain and swelling of four months duration over proximal and lateral aspect of right leg. Pain was dull aching, progressive, aggravated by activity and relieved by rest. Swelling was noted by patient incidentally. It gradually progressed to attain its present size of $3 \times 4 \mathrm{~cm}$. Overlying skin was normal in appearance and mobile. The patient denied any history of recent trauma to the extremity and reported no respiratory or systemic illness preceding the leg pain. He reported no fever, chills, or other constitutional symptoms and had screened negative for the human immunodeficiency virus (HIV) testing. There was no other history suggestive of immuno-compromised status.

Physical examination revealed that the patient was afebrile and that the vital signs were within normal limits .Examination of the right knee joint revealed swelling laterally over fibular head, $3 \times 4 \mathrm{~cm}$. in dimension, globular sized, tender, fixed to bone, hard in consistency. Swelling was not adherent to skin and but was fixed to bone. Patient had full range of motion at knee joint without distal neurovascular deficit. Provisional diagnosis of case was aneurysmal bone cyst or giant cell tumour.

All hematological investigation was normal including the CD4 count. Routine antero-posterior X-ray of knee [Figure-2a] showed a single lytic expansive lesion at proximal fibula in epiphyseo-metaphyseal region with cortical thinning and narrow zone of transition in mature skeleton. $\mathrm{X}$ ray chest was normal. MRI of leg [Figure-1] showed an aggressive neoplastic lesion with cortical breech and large soft tissue component with invasion of lateral tibial plateau with significant soft tissue edema suggestive of GCT or clear cell chondrosarcoma. FNAC failed to give definitive diagnosis. So decision was taken to excise the tumor, during excisional biopsy [Figure-2b] an incision was taken over lateral aspect of upper leg and lateral popliteal nerve was isolated. Intraoperative findings were of a mass with a rough surface and blackish scattered circular areas over whole of the mass .Then the mass was excised en-block. Tibial condyle was found involved and curetted, hemostasis was achieved and wound closed meticulously over drain. The excised mass was sent for histopathology and cytology examination [Figures-3a and 3b] which showed large fungal balls surrounded by chronic granulomatous infiltrate of inflammatory cells with foreign body giant cells. No neoplastic lesion seen. Histopathology suggested fungal granulomatous lesion most probably caused by Aspergellus fumigatus. Post- 
operative antibiotics and antifungal agent (Amphotericin B) were given. The wound healed without any complications and sutures were removed on day fifteen.

\section{Discussion}

Fungal osteomyelitis of long bone in immune-competent host has been reported very rarely in literature [3,4]. Presentation may be asymptomatic, acute or chronic pain, diffuse swelling or in the form of sinuses or ulcers. [5] Because of their comparative rarity and resemblance, clinically and roentgenographically, to more common infections, they are readily overlooked. [6] Even on culture, the report is frequently "staphylococci" or "nogrowth". The definitive documentation of bone infection due to an ordinary, ubiquitous saprophytic fungus requires three procedures: (i) cultural isolation and accurate identification of the organisms, preferably repeated, from the actual site of infection; (ii) direct microscopic demonstration of the pathogenic invasive form of the fungus in material from the site where the cultures were obtained (a pus smear or histologic section of the tissue); and (iii) correlation of the results of the culture to the osteomyelitic process [7]. In this case we had confirmed our diagnosis by this method.

\section{Conclusion-:}

In most cases of fungal osteomyelitis, gross defects in immune-competence are present. Among children, chronic granulomatous disease is the most common defect, and among adults, immunosuppressive drug therapy is the most important predisposing condition [3,8]. In healthy individuals the skin and mucous membranes are first-line barriers against infections, but these barriers are regularly violated by minor or major trauma or surgical procedures. The common areas of fungal involvement are brain and lungs having candida and aspergillus as most common pathogens. Fungal osteoarthritis and osteomyelitis following surgical procedures or trauma have been occasionally described [9]. These infections can present as joint effusion, arthritis, osteolytic lesions and osteomyelitis.[5]Large tubular bones are less commonly involved. Many times granulomatous fungal infection presenting as lytic lesion on radiograph may mimic bone tumours such as aneurysmal bone cyst, enchondroma or a giant cell tumour $[10,11,12,13]$ and diagnosis frequently comes as a surprise by recognition of the organism by histopathological examination. However the diagnosis needs to be confirmed by culture studies. Therefore diagnosis of fungal infections requires a high index of suspicion. The text also emphasizes the importance of including endemic fungal infections in the differential diagnosis of bone lesions.

Clinical message: Fungal infections are uncommon in immune-competent host. Diagnosis of fungal infections requires a high index of suspicion and needs to be confirmed by culture studies. Fungal infections should be included in differential diagnosis of bone lesions in endemic areas.

- Consent-

The patient had given informed consent in written for possible publication of his findings.

- Competing interests

NIL

- Acknowedgements

NIL

\section{References}

[1]. Cohen M, Bonfiglio, Campbell C J. Orthopedic pathophysiology in diagnosis and treatment. New York, N.Y: Churchill Livingstone; 1996. Inflammation and infection; pp. 19-52.

[2]. Casscells S W. Aspergillus osteomyelitis of the tibia. J Bone Joint Surg. 1978;60:994-995.

[3]. De Vuyst D, Surmont I, Verhaegen J, Vanhaecke J. Tibial osteomyelitis due to Aspergillus flavus in heart transplant patient. Infection. 1992;20:48-49.

[4]. De Hoog G S, Guarro J, editors. Atlas of clinical fungi. Baarn, The Netherlands: Centraalbureau voor Shimmelcultures/Universitat Rovira I Virgili; 1995.

[5]. Lucas morretti et al radiological findings of osteoarticular infection in paracoccidiomycosis. Skeletal .Radiol .2011; 18 may

[6]. Ray A. Carter infectious granuloma of bones and joints, with Special Reference to Coccidioidal Granuloma . Radiology; 1934(7), 23: 1-16

[7]. Simpson M B, Merz W G, Kurlinski J P, Solomon M. Opportunistic mycotic osteomyelitis: bone infection due to Aspergillus and Candida species. Medicine. 1977;56:475-482.

[8]. Tack K J, Rhame F S, Brown B, Thompson R C. Aspergillus osteomyelitis. Report of four cases and review of the literature. Am J Med. 1982;73:295-300.

[9]. G P, Anaissie E J. Opportunistic infections: a major problem in immunocompromised patients. In: Richardson R G, editor. Opportunistic fungal infections: focus on fluconazole. International Congress and Symposium series 153. London, United Kingdom: Royal Society of Medicine; 1989. pp. 1-16.

[10]. J. I. HUANG Coccidioidomycosis Fungal Infection in the Hand Mimicking a Metacarpal Enchondroma .J Hand Surg Eur.2000;1 25:475-477

[11]. The Journal Of Bone And Joint Surgery 4 May 2011 : case report of 33 year old man presenting with wrist fungal granuloma. 
[12]. Fungal infection by Paracoccidiodes brasiliensis micking bone tumour: Pediatric Blood Cancer, Volume 50,Issue6 pages 12841286 June 2008

[13]. Clues to recognition of fungal origin of lytic skeletal lesions. I Hershkovitz, B M Rothschild, O Dutour, C Greenwald; Am J Phys Anthropol 1998 May;106(1):47-60

\section{Figures}

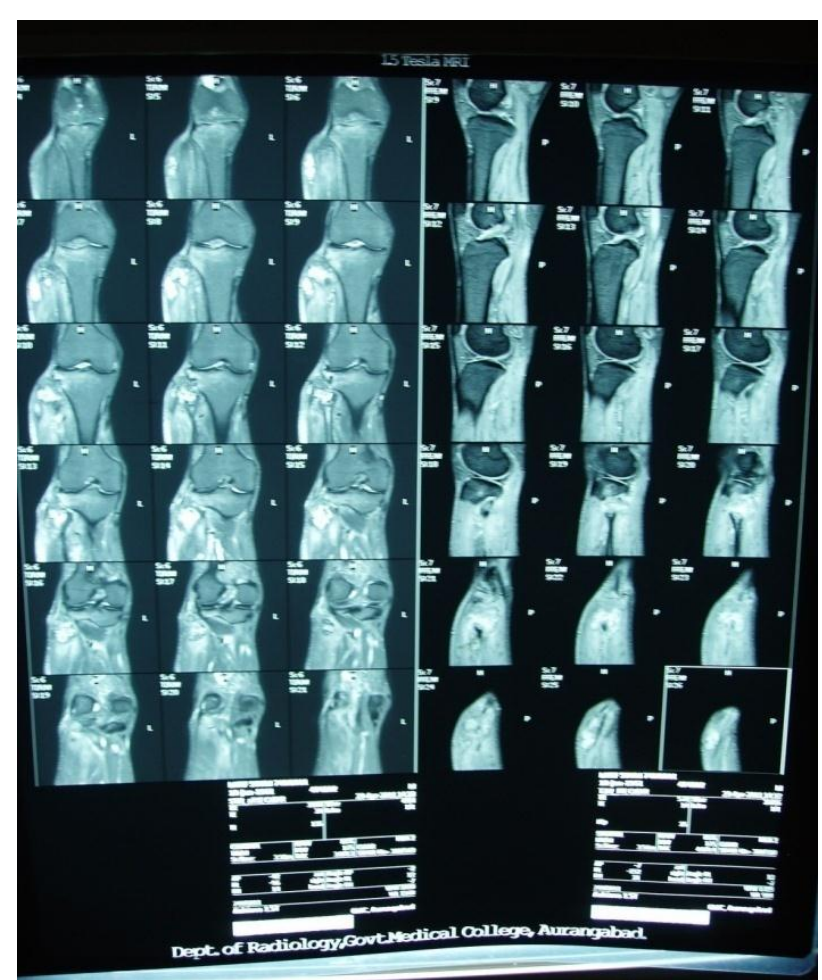

Figure 1

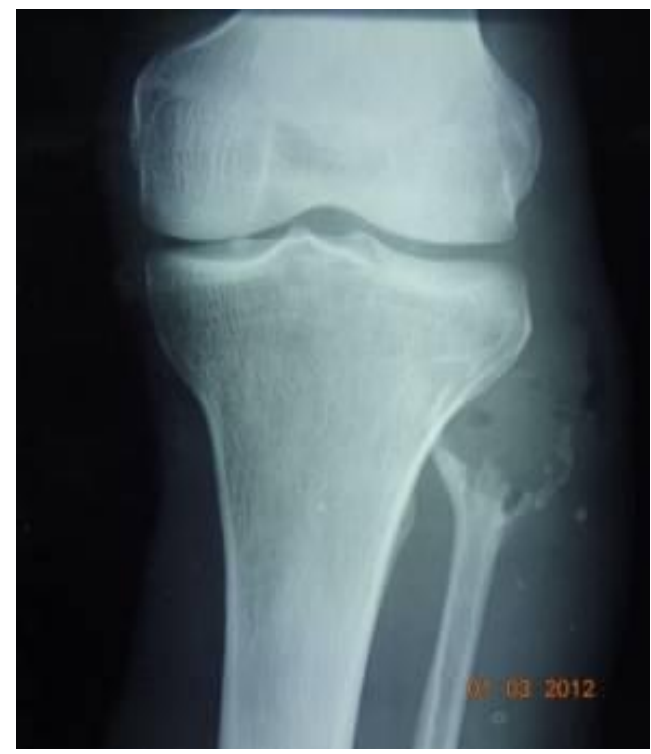

Figure 2a 


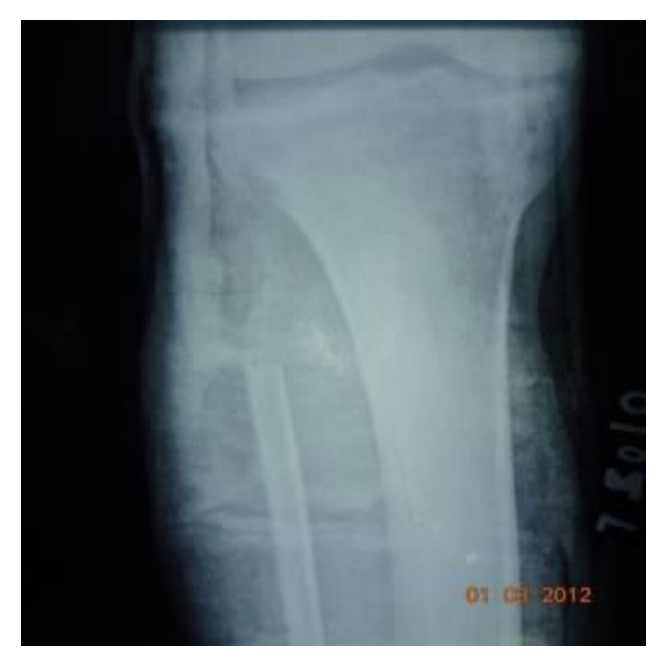

Figs-2b:



Figure 3a

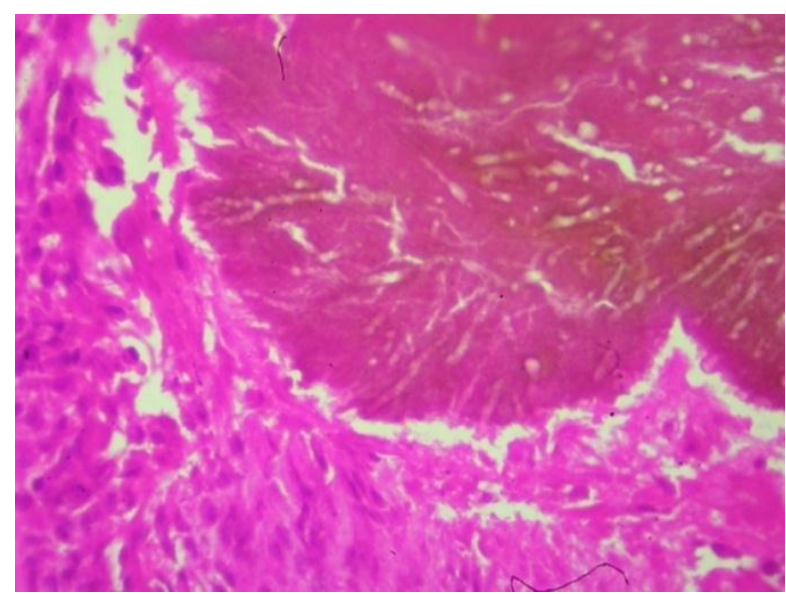

Figs-3b

Figure Legends

Figure 1--MRI : RIGHT KNEE JOINT SHOWING LESION PROXIMAL FIBULA

Figure 2a-Preoperative X-Ray With Lytic Lesion In Head Of Fibula

Figure 2b--Postoperative X-Ray After Excision Of Mass With Fibular Head

Figure 3a And 3b--Histopathology Slides. 